\title{
Existence of positive solutions for an approximation of stationary mean-field games
}

Nojood Almayouf $^{1}$, Elena Bachini ${ }^{2}$, Andreia Chapouto ${ }^{3}$, Rita Ferreira ${ }^{4}$, Diogo Gomes $^{5}$, Daniela Jordão ${ }^{6}$, David Evangelista Junior ${ }^{7}$, Avetik Karagulyan $^{8}$, Juan Monasterio ${ }^{9}$, Levon Nurbekyan ${ }^{10}$, Giorgia Pagliar ${ }^{11}$, Marco Piccirilli ${ }^{12}$, Sagar Pratapsi ${ }^{13}$, Mariana Prazeres ${ }^{14}$, João Reis ${ }^{15}$, André Rodrigues $^{16}$, Orlando Romero ${ }^{17}$, Maria Sargsyan ${ }^{18}$, Tommaso Seneci ${ }^{19}$, Chuliang Song ${ }^{20}$, Kengo Terai ${ }^{21}$, Ryota Tomisaki ${ }^{22}$, Hector Velasco-Perez ${ }^{23}$, Vardan Voskanyan ${ }^{24}$, and Xianjin Yang ${ }^{25}$

${ }^{1}$ Effat University, Kingdom of Saudi Arabia

${ }^{2}$ University of Padova, Italy

${ }^{3}$ University of Coimbra, Portugal

${ }^{4}$ KAUST, Kingdom of Saudi Arabia

${ }^{5}$ KAUST, Kingdom of Saudi Arabia

${ }^{6}$ University of Coimbra, Portugal

${ }^{7}$ KAUST, Kingdom of Saudi Arabia

${ }^{8}$ Yerevan State University, Armenia

${ }^{9}$ University of Buenos Aires, Argentina

${ }^{10}$ KAUST, Kingdom of Saudi Arabia

${ }^{11}$ University of Verona, Italy

${ }^{12}$ University of Rome Tor Vergata, Italy

${ }^{13}$ University of Coimbra, Portugal

${ }^{14}$ KAUST, Kingdom of Saudi Arabia

${ }^{15}$ University of Lisbon, Portugal

${ }^{16}$ University of Coimbra, Portugal

${ }^{17}$ University of Lisbon, Portugal

${ }^{18}$ American University of Armenia, Armenia

${ }^{19}$ University of Verona, Italy

${ }^{20}$ Zhejiang University, China

${ }^{21}$ Waseda University, Japan

${ }^{22}$ Waseda University, Japan

${ }^{23}$ National Autonomous University of Mexico, Mexico

${ }^{24}$ KAUST, Kingdom of Saudi Arabia

${ }^{25}$ KAUST, Kingdom of Saudi Arabia

December 15, 2015

\begin{abstract}
Here, we consider a regularized mean-field game model that features a low-order regularization. We prove the existence of solutions with positive density. To do so, we combine a priori estimates with the continuation method. In contrast with highorder regularizations, the low-order regularizations are easier to implement numerically. Moreover, our methods give a theoretical foundation for this approach.
\end{abstract}


Rita Ferreira, Diogo Gomes, David Evangelista Junior, Levon Nurbekyan, Mariana Prazeres, Vardan Voskanyan, and Xianjin Yang were partially supported by KAUST baseline and start-up funds and KAUST SRI, Uncertainty Quantification Center in Computational Science and Engineering. The other authors were partially supported by KAUST Visiting Students Research Program.

\section{Prologue}

On August 22, 2015, eighteen young mathematicians (B.Sc. and M.Sc. Students) arrived at King Abdullah University of Science and Technology (KAUST) in Thuwal, Kingdom of Saudi Arabia. They were participants in the first KAUST summer camp in Applied Partial Differential Equations. Among them were Argentinians, Armenians, Chinese, Italians, Japanese, Mexicans, Portuguese, and Saudis. For many of them, this was their first time abroad. All were looking forward to the following three weeks.

We designed the summer camp to give an intense hands-on three-week Ph.D. experience. It comprised courses, seminars, a project, and a final presentation. The project was an essential component of the summer camp, and its main outcome is the present paper. Our objectives were to introduce students to an active research topic, teach effective paper writing techniques, and develop their presentation skills. Numerous challenges lay ahead. First, we had three weeks to achieve these goals. Second, students had distinct backgrounds. Third, we planned to study a research-level problem, not a simple exercise.

We selected a problem in mean-field games, a recent and active area of research. The primary goal was to prove the existence of solutions of a system of partial differential equations. To avoid unnecessary technicalities, we considered the one-dimensional case, where the partial differential equations become ordinary differential equations. The project involved partial differential equation methods that are usually taught in advanced courses: a priori estimate methods, the infinite dimensional implicit function theorem, and the continuation method. In spite of the elementary nature of the proofs, the results presented here are a relevant and original contribution to the theory of mean-field games.

We divided the students into five groups and assigned tasks to each of them. Roughly, each of the sections of this paper corresponds to a task. The students were given a rough statement of the results to be proven, and their task was to figure out the appropriate assumptions, the precise statements, and the proofs. The work of the different groups had to be coordinated to make sure that the assumptions, results, and proofs fit nicely with each other and that duplicate work was avoided. Several KAUST graduate students and post-docs were of invaluable help in this regard.

This project would not have been possible within such a short time frame without the use of new technologies. The paper was written in a collaborative fashion using the platform http://authorea.com that allowed all the groups to work simultaneously. In this way, all groups had access to the latest version of the assumptions and to the current statements of the theorems and propositions. Each group could easily comment and make corrections on other group's work.

This project illustrates how research in mathematics can be a collaborative experience even with a large number of participants. Moreover, it gave each of the students in the summer camp a glimpse of real research in mathematics. Finally, this was the first experience for the Ph.D. students and post-docs who helped in this project in mentoring and advising students. This summer camp was a unique and valuable experience for all participants whose results we share in this paper.

\section{Introduction}

Mean-field game (MFG) theory is the study of strategic decision making in large populations of small interacting individuals who are also called agents or players. The MFG framework was developed in the engineering community by Caines, Huang, and Malhamé [18, 19] and in the mathematical community by Lasry and Lions [20, 21, 22] (also see 23]). These games 
model the behavior of rational agents who play symmetric differential games. In these problems, each player chooses their optimal strategy in view of global (or macroscopic) statistical information on the ensemble of players. This approach leads to novel problems in nonlinear equations. Current research topics are the applications of MFGs (including, for example, growth theory in economics and environmental policy), mathematical problems related to MFGs (existence, uniqueness, and regularity questions), and numerical methods in the MFGs framework (discretization, convergence, and efficient implementation).

Here, we consider the following problem:

Problem 1. Let $\mathbb{T}=\mathbb{R} / \mathbb{Z}$ denote the one-dimensional torus, identified with the interval $[0,1]$ whenever convenient. Fix a $C^{2}$ Hamiltonian, $H: \mathbb{R} \rightarrow \mathbb{R}$, and a continuous potential, $V: \mathbb{T} \rightarrow \mathbb{R}$. Let $\alpha$ and $\epsilon$ be positive numbers with $\epsilon \leqslant 1$ for definedness. Find $u, m \in C^{2}(\mathbb{T})$ satisfying $m>0$ and

$$
\left\{\begin{array}{l}
u-u_{x x}+H\left(u_{x}\right)+V(x)=m^{\alpha}+\epsilon\left(m-m_{x x}\right) \\
m-m_{x x}-\left(H^{\prime}\left(u_{x}\right) m\right)_{x}=1-\epsilon\left(u-u_{x x}\right) .
\end{array}\right.
$$

In this problem, $m$ is the distribution of players and $u(x)$ is the value function for a typical player in the state $x$. We stress that the condition $m>0$ is an essential component of the problem. So, if $(u, m)$ solves the Problem 11, we require $m$ to be strictly positive. We will show the existence of solutions to this problem under suitable assumptions on the Hamiltonian that are described in Section 3. An example that satisfies those assumptions is $H(p)=\left(1+p^{2}\right)^{\gamma / 2}$ with $1<\gamma<2$, and any $V: \mathbb{T} \rightarrow \mathbb{R}$ of class $C^{2}$.

When $\epsilon=0$, 2.1) becomes

$$
\left\{\begin{array}{l}
u-u_{x x}+H\left(u_{x}\right)+V(x)=m^{\alpha} \\
m-m_{x x}-\left(H^{\prime}\left(u_{x}\right) m\right)_{x}=1 .
\end{array}\right.
$$

The system in (2.2) is a typical MFG model similar to the one introduced in 20]. The Legendre transform of the Hamiltonian, $H$, given by $L(v)=\sup _{p}-p v-H(p)$ is the cost in units of time that an agent incurs by choosing to move with a drift $v$; the potential, $V$, accounts for spatial preferences of the agents; the term $m^{\alpha}$ encodes congestion effects.

The MFG models proposed in [20, 21] consist of a system of partial differential equations that have (2.2) as a particular case. The current literature covers a broad range of problems, including stationary problems [8, 13, 14, 15, 25, heterogeneous populations [2, time-dependent models $[1,9,10,11,12,26,27$, congestion problems [6, 17, and obstacletype problems [7]. For a recent account of the theory of MFG, we suggest the survey paper [16] and the courses [23] and 24].

The system in (2.1) arises as an approximation of (2.2) that preserves monotonicity properties. Monotonicity-preserving approximations to MFG systems were introduced in [5]. In that paper, the authors consider mean-field games in dimension $d \geqslant 1$ that include the following example:

$$
\left\{\begin{array}{l}
u-\Delta u+H(D u, x)+V(x)=m^{\alpha}+\epsilon\left(m+\Delta^{2 q} m\right)+\beta_{\epsilon}(m) \\
m-\Delta m-\operatorname{div}\left(D_{p} H(D u, x) m\right)=1-\epsilon\left(u+\Delta^{2 q} u\right)
\end{array}\right.
$$

where $q$ is a large enough integer, and $\beta_{\epsilon}$ is a suitable penalization that satisfies $\beta_{\epsilon}(m) \rightarrow-\infty$ as $m \rightarrow 0$. Then, as $\epsilon \rightarrow 0$, the solutions of (2.3) converge to solutions of (2.2). Yet, from the perspective of numerical methods, both the high-order degree of (2.3) and the singularity caused by the penalty, $\beta_{\epsilon}$, are unsatisfactory due to a poor conditioning of discretizations. Here, we investigate a low-order regularization that may be more suitable for computational problems.

A fundamental difficulty in the analysis of (2.1) is the non-negativity of $m$. The FokkerPlanck equation in (2.2) has a maximum principle, and, consequently, $m \geqslant 0$ for any solution of (2.2). Due to the coupling, this property is not evident in the corresponding equation in (2.1). The previous regularization in (2.3) relies on a penalty that forces the positivity of 
$m$. This mechanism does not exist in (2.1), and we are not aware of any general method to prove the existence of positive solutions of (2.1).

Our main result is the following theorem:

Theorem 2.1. Suppose Assumptions 17 hold (cf. Section 多). Then, there exists $\epsilon_{0}>0$ such that for all $0<\epsilon<\epsilon_{0}$, Problem 1 admits a $C^{2, \frac{1}{2}}$ solution $(u, m)$.

Theorem 2.1 introduces a low-order regularization procedure for (2.2) for which existence of solutions can be established without penalty terms. Because high-order regularization methods and penalty terms create serious difficulties in the numerical implementation, this result is relevant to the numerical approximation of (2.2). Moreover, we believe that the techniques we consider here can be extended to higher-dimensional problems.

To prove the main result, we use the continuation method. The first step is to establish a priori estimates for the solutions of (2.1). Then, we replace the potential, $V$, by $\lambda V$ for $0 \leqslant \lambda \leqslant 1$. For $\lambda=0$, which corresponds to $V=0$ in (2.1), we determine an explicit solution. The a priori estimates give that the set, $\Lambda$, of values, $\lambda$, for which (2.1) has a solution is a closed set. Finally, we apply an infinite-dimensional version of the implicit function theorem to show that $\Lambda$ is relatively open in $[0,1]$. This proves the existence of solutions.

The remainder of this paper is structured as follows. We discuss the main assumptions in Section 3. Next, in Section 4, we start our study of (2.1) by considering the case $V=0$ and constructing an explicit solution. Sections 5 9 are devoted to a priori estimates for solutions of (2.1). These estimates include energy and second-order bounds, discussed respectively in Sections 5 and 6. Hölder and $C^{2, \frac{1}{2}}$ estimates, addressed respectively in Sections 7 and 8 , and lower bounds on $m$, given in Section 9. Next, we lay out the main results needed for the implicit function theorem. We introduce the linearized operator in Section 10 and discuss its injectivity and surjectivity properties. Finally, the proof of Theorem 2.1 is presented in Section 11

\section{Main Assumptions}

To prove Theorem 2.1] we need to introduce various assumptions that are natural in this class of problems. These encode distinct properties of the Hamiltonian in a convenient way. We begin by stating a polynomial growth condition for the Hamiltonian.

Assumption 1. There exist positive constants, $C_{1}, C_{2}, C_{3}$, and $\gamma>1$, such that for all $p \in \mathbb{R}$, the Hamiltonian, $H$, satisfies

$$
-C_{1}+C_{2}|p|^{\gamma} \leqslant H(p) \leqslant C_{1}+C_{3}|p|^{\gamma} .
$$

For convex Hamiltonians, the expression $p H^{\prime}(p)-H(p)$ is the Lagrangian written in momentum coordinates. The next assumption imposes polynomial growth in this quantity.

Assumption 2. There exist positive constants, $\tilde{C}_{1}, \tilde{C}_{2}$, and $\tilde{C}_{3}$, such that for all $p \in \mathbb{R}$, we have

$$
-\tilde{C}_{1}+\tilde{C}_{2}|p|^{\gamma} \leqslant p H^{\prime}(p)-H(p) \leqslant \tilde{C}_{1}+\tilde{C}_{3}|p|^{\gamma} .
$$

Because we look for solutions $(u, m) \in C^{2, \frac{1}{2}}(\mathbb{T}) \times C^{2, \frac{1}{2}}(\mathbb{T})$ of Problem 1 we require in Assumption 3 and Assumption 5 more regularity for $V$ and $H$.

Assumption 3. The potential, $V$, is of class $C^{2}$.

Because the Hamilton-Jacobi equation in (2.2) arises from an optimal control problem, it is natural to suppose that the Hamiltonian, $H$, is convex.

Assumption 4. $H$ is convex. 
Assumption 5. The Hamiltonian, $H$, is of class $C^{4}$.

Here, we work with subquadratic Hamiltonians. Accordingly, we impose the following condition on $\gamma$.

Assumption 6. $\gamma<2$.

Finally, we state a growth condition on the derivative of the Hamiltonian. The exponent, $\gamma$, is the same as in Assumptions 1 1 and 2 This is a natural growth condition that the model $H(p)=\left(1+|p|^{2}\right)^{\frac{\gamma}{2}}$ satisfies.

Assumption 7. There exists a positive constant, $\bar{C}$, such that for all $p \in \mathbb{R}$, we have

$$
\left|H^{\prime}(p)\right| \leqslant \bar{C}\left(1+|p|^{\gamma-1}\right) .
$$

\section{The $V=0$ case}

To prove Theorem 2.1 we use the continuation method. More precisely, we consider system (2.1) with $V$ replaced by $\lambda V$ for $0 \leqslant \lambda \leqslant 1$. Next, we show the existence of the solution for all $0 \leqslant \lambda \leqslant 1$. As a starting point, we study the $\lambda=0$ case; that is, $V=0$. We show that (2.1) admits a solution in this particular instance.

Proposition 4.1. Suppose that $V=0$. Then, there exists an $\epsilon_{0}>0$ such that for all $0<\epsilon<\epsilon_{0}$, Problem 1 admits a solution $(u, m)$.

Proof. We look for constant solutions $(u, m)$. In this case, we have $u_{x}=u_{x x}=m_{x}=m_{x x}=$ 0 . Accordingly, (2.1) reduces to

$$
\left\{\begin{array}{l}
u+H(0)=m^{\alpha}+\epsilon m \\
m=1-\epsilon u .
\end{array}\right.
$$

In the previous system, solving the first equation for $u$ and replacing the resulting expression into the second, we get

$$
\epsilon m^{\alpha}+\left(1+\epsilon^{2}\right) m-1-\epsilon H(0)=0 .
$$

We set $g(m)=\epsilon m^{\alpha}+\left(1+\epsilon^{2}\right) m-1-\epsilon H(0)$, so that (4.1) reads $g(m)=0$. Next, we notice that $g(0)=-1-\epsilon H(0)$. For small enough $\epsilon_{0}>0$ and for all $0<\epsilon<\epsilon_{0}$, we have $g(0)<0$. On the other hand, if we take a constant $C>|H(0)|$, we have

$$
g(1+\epsilon C)>1+\epsilon C-1-\epsilon H(0)=\epsilon(C-H(0))>0 .
$$

Because $0<1+\epsilon C$, by the intermediate value theorem, there exists a constant $m_{0} \in$ ] $0,1+\epsilon C\left[\right.$ such that $g\left(m_{0}\right)=0$. Then, setting $u_{0}=\left(1-m_{0}\right) / \epsilon$, we conclude that the pair $\left(u_{0}, m_{0}\right)$ satisfies the requirements.

Remark 4.2. Note that if $H(0)>0$, then $g(0)<0$ and $g(1+\epsilon C)>0$. In this case, the previous proposition holds for all $\epsilon>0$.

\section{$5 \quad$ Energy estimates}

MFG systems such as (2.2) admit many a priori estimates. Among those, energy estimates stand out for their elementary proof - the multiplier method. Here, we apply this method to (2.1). 
Proposition 5.1. Suppose that Assumptions 1 and 2 hold. Let $(u, m)$ solve Problem 1 . Then,

$$
\int_{0}^{1} m^{\alpha+1} d x+\int_{0}^{1}\left|u_{x}\right|^{\gamma}(1+m) d x+\epsilon \int_{0}^{1}\left(u^{2}+m^{2}+u_{x}^{2}+m_{x}^{2}\right) d x \leqslant C,
$$

where $C$ is a universal positive constant depending only on the constants in Assumptions 1 and 国 and on $\|V\|_{L^{\infty}}$.

Proof. We begin by multiplying the first equation in (2.1) by $(1+\epsilon-m)$ and the second one by $u$. Adding the resulting expressions and integrating, we get

$$
\begin{gathered}
\int_{0}^{1}\left[(1+\epsilon) H\left(u_{x}\right)+m\left(u_{x} H^{\prime}\left(u_{x}\right)-H\left(u_{x}\right)\right)\right] d x+\int_{0}^{1} m^{\alpha+1} d x+\epsilon \int_{0}^{1}\left(u^{2}+m^{2}+u_{x}^{2}+m_{x}^{2}\right) d x \\
=-\epsilon \int_{0}^{1} u d x+\int_{0}^{1}(m-1-\epsilon) V(x) d x+(1+\epsilon) \int_{0}^{1} m^{\alpha} d x+\epsilon(1+\epsilon) \int_{0}^{1} m d x, \quad \text { (5.2) }
\end{gathered}
$$

where we also used integration by parts and the periodicity of $u$ and $m$ to obtain

$$
\begin{gathered}
\int_{0}^{1} m u_{x x} d x-\int_{0}^{1} u m_{x x} d x=0 \\
\int_{0}^{1} u_{x x} d x=\left.u_{x}\right|_{0} ^{1}=0, \quad \int_{0}^{1} m_{x x} d x=\left.m_{x}\right|_{0} ^{1}=0 \\
\int_{0}^{1} m m_{x x} d x=-\int_{0}^{1} m_{x}^{2} d x, \quad \int_{0}^{1} u u_{x x} d x=-\int_{0}^{1} u_{x}^{2} d x
\end{gathered}
$$

and

$$
\int_{0}^{1} u\left(H^{\prime}\left(u_{x}\right) m\right)_{x} d x=-\int_{0}^{1} u_{x} H^{\prime}\left(u_{x}\right) m d x
$$

Next, we observe that by Assumptions 1 and 2, and using the fact that $0<\epsilon \leqslant 1$, we have

$$
\begin{gathered}
\int_{0}^{1}\left[(1+\epsilon) H\left(u_{x}\right)+m\left(H^{\prime}\left(u_{x}\right) u_{x}-H\left(u_{x}\right)\right)\right] d x \\
\geqslant \int_{0}^{1}\left[-2 C_{1}-\tilde{C}_{1} m+K_{0}\left|u_{x}\right|^{\gamma}(1+m)\right] d x,
\end{gathered}
$$

where $K_{0}:=\min \left\{C_{2}, \tilde{C}_{2}\right\}$.

From (5.2) and (5.3), it follows that

$$
\begin{aligned}
& \int_{0}^{1} K_{0}\left|u_{x}\right|^{\gamma}(1+m) d x+\int_{0}^{1} m^{\alpha+1} d x+\epsilon \int_{0}^{1}\left(u^{2}+m^{2}+u_{x}^{2}+m_{x}^{2}\right) d x \\
& \quad \leqslant \frac{\epsilon}{2} \int_{0}^{1} u^{2} d x+\frac{1}{2}+\left(\|V\|_{\infty}+2+\tilde{C}_{1}\right) \int_{0}^{1} m d x+2 \int_{0}^{1} m^{\alpha} d x+2\left(\|V\|_{\infty}+C_{1}\right),
\end{aligned}
$$

where we also used the estimates $2 u \leqslant u^{2}+1$ and $0<\epsilon \leqslant 1$.

Finally, we observe that for every $\delta_{1}, \delta_{2}>0$, there exist constants, $K_{1}$ and $K_{2}$, such that

$$
\int_{0}^{1} m^{\alpha} d x \leqslant \delta_{1} \int_{0}^{1} m^{\alpha+1} d x+K_{1}, \quad \int_{0}^{1} m d x \leqslant \delta_{2} \int_{0}^{1} m^{\alpha+1} d x+K_{2}
$$

Consequently, taking $\delta_{1}=\frac{1}{8}$ and $\delta_{2}=\frac{1}{4\left(\|V\|_{\infty}+2+\tilde{C}_{1}\right)}$ in (5.5) and using the resulting estimates in (5.4), we conclude that (5.1) holds.

Corollary 5.2. Suppose that Assumptions 1 and a hold. Let $(u, m)$ solve Problem 1 , Then,

$$
\int_{0}^{1} m d x \leqslant C
$$

where $C$ is a universal positive constant depending only on the constants in Assumptions 1 and 圆 and on $\|V\|_{L^{\infty}}$. 
Proof. Due to (5.1) and because $m$ is positive,

$$
\int_{0}^{1} m^{\alpha+1} \leqslant C
$$

where $C$ is a universal positive constant depending only on the constants in Assumptions 1 and 2 and on $\|V\|_{L^{\infty}}$. Consequently, using Young's inequality, we have that

$$
\int_{0}^{1} m d x \leqslant \frac{1}{\alpha+1} \int_{0}^{1} m^{\alpha+1} d x+\frac{\alpha}{\alpha+1} \leqslant \frac{C}{\alpha+1}+\frac{\alpha}{\alpha+1} .
$$

\section{Second-order estimates}

We proceed in our study of (2.1) by examining another technique to obtain a priori estimates. These estimates give additional control over high-order norms of the solutions.

Proposition 6.1. Suppose that Assumption 圆 holds. Let $(u, m)$ solve Problem 1. Then, we have

$$
\int_{0}^{1}\left(H^{\prime \prime}\left(u_{x}\right) u_{x x}^{2} m+\alpha m^{\alpha-1} m_{x}^{2}\right) d x+\epsilon \int_{0}^{1}\left(m_{x}^{2}+m_{x x}^{2}+u_{x}^{2}+u_{x x}^{2}\right) d x \leqslant C,
$$

where $C>0$ denotes a universal constant depending only on $\|V\|_{C^{2}}$. Moreover, under Assumption 4

$$
\int_{0}^{1} \alpha m^{\alpha-1} m_{x}^{2} d x+\epsilon \int_{0}^{1}\left(m_{x}^{2}+m_{x x}^{2}+u_{x}^{2}+u_{x x}^{2}\right) d x \leqslant C .
$$

Proof. To simplify the notation, we represent by $C$ any positive constant that depends only on $\|V\|_{C^{2}}$ and whose value may change from one instance to another.

Multiplying the first equation in (2.1) by $m_{x x}$ and the second one by $u_{x x}$ yields

$$
\begin{aligned}
& \left(u-u_{x x}+H\left(u_{x}\right)+V(x)\right) m_{x x}=\left(m^{\alpha}+\epsilon\left(m-m_{x x}\right)\right) m_{x x}, \\
& \left(m-m_{x x}-\left(H^{\prime}\left(u_{x}\right) m\right)_{x}\right) u_{x x}=\left(1-\epsilon\left(u-u_{x x}\right) u_{x x} .\right.
\end{aligned}
$$

Subtracting the above equations integrated over $[0,1]$ gives

$$
\begin{aligned}
& \int_{0}^{1}\left(u m_{x x}-m u_{x x}+u_{x x}\right) d x+\int_{0}^{1}\left(H\left(u_{x}\right) m_{x x}+\left(H^{\prime}\left(u_{x}\right) m\right)_{x} u_{x x}\right) d x \\
& \quad+\int_{0}^{1} V(x) m_{x x} d x-\int_{0}^{1} m^{\alpha} m_{x x} d x+\epsilon \int_{0}^{1}\left(-m m_{x x}+m_{x x}^{2}-u u_{x x}+u_{x x}^{2}\right) d x=0 .
\end{aligned}
$$

Next, we evaluate each of the integrals above. Using the integration by parts formula and the periodicity of boundary conditions, we have

$$
\int_{0}^{1}\left(u m_{x x}-m u_{x x}+u_{x x}\right) d x=0
$$

In addition,

$$
\begin{aligned}
& \int_{0}^{1}\left[\left(H^{\prime}\left(u_{x}\right) m\right)_{x} u_{x x}+H\left(u_{x}\right) m_{x x}\right] d x \\
& \quad=\int_{0}^{1}\left[H^{\prime \prime}\left(u_{x}\right) m u_{x x}^{2}+\left(H\left(u_{x}\right)\right)_{x} m_{x}+\left(H\left(u_{x}\right)\right) m_{x x}\right] d x=\int_{0}^{1} H^{\prime \prime}\left(u_{x}\right) m u_{x x}^{2} d x .
\end{aligned}
$$

Furthermore, we have

$$
-\int_{0}^{1} m^{\alpha} m_{x x} d x=\int_{0}^{1} \alpha m^{\alpha-1} m_{x}^{2} d x
$$


and

$$
\int_{0}^{1}-V m_{x x} d x=-\int_{0}^{1} V_{x x} m d x \leqslant \int_{0}^{1}\left|V_{x x}\right| m d x \leqslant C \int_{0}^{1} m d x \leqslant C,
$$

where we used Corollary 5.2

Finally,

$$
\epsilon \int_{0}^{1}\left(-m m_{x x}+m_{x x}^{2}-u u_{x x}+u_{x x}^{2}\right) d x=\epsilon \int_{0}^{1}\left(m_{x}^{2}+m_{x x}^{2}+u_{x}^{2}+u_{x x}^{2}\right) \mathrm{d} x .
$$

Using (6.3)- -6.8$)$, we get

$$
\begin{aligned}
& \int_{0}^{1} H^{\prime \prime}\left(u_{x}\right) m u_{x x}^{2} \mathrm{~d} x+\int_{0}^{1} \alpha m^{\alpha-1} m_{x}^{2} \mathrm{~d} x \\
& \quad+\epsilon \int_{0}^{1}\left(m_{x}^{2}+m_{x x}^{2}+u_{x}^{2}+u_{x x}^{2}\right) \mathrm{d} x=-\int_{0}^{1} V m_{x x} \leqslant C .
\end{aligned}
$$

This completes the proof of (6.1). To conclude the proof of Proposition 6.1, we observe that Assumption 4 implies that $H^{\prime \prime}$ is a non-negative function, which together with (6.1) gives (6.2).

\section{$7 \quad$ Hölder continuity}

We recall that Morrey's theorem in one-dimension 4 gives the following result.

Proposition 7.1. Let $f \in C^{1}(\mathbb{T})$. Then,

$$
|f(x)-f(y)| \leqslant\left\|f_{x}\right\|_{L^{2}}|x-y|^{\frac{1}{2}}, \quad \forall x, y \in \mathbb{T} .
$$

Proposition 7.2. Suppose that Assumptions 14 hold. Let $(u, m)$ solve Problem 1 . Then, $u, u_{x}, m$, and $m_{x}$ are $\frac{1}{2}$-Hölder continuous functions with $L^{\infty}$-norms and Hölder constants bounded by $\frac{C}{\sqrt{\epsilon}}$, where $C$ is a universal constant depending only on the constants in Assumptions 1 and 2 and on $\|V\|_{C^{2}}$.

Proof. By Proposition 5.1] we have that

$$
\epsilon \int_{0}^{1}\left(m^{2}+u^{2}+m_{x}^{2}+u_{x}^{2}\right) d x \leqslant C
$$

where $C$ is a universal constant depending only on the constants in Assumptions 1 and 2 and on $\|V\|_{L^{\infty}}$.

According to Proposition 7.1 we have

$$
|u(x)-u(y)| \leqslant\left\|u_{x}\right\|_{L^{2}}|x-y|^{\frac{1}{2}}, \quad \forall x, y \in \mathbb{T} .
$$

Moreover, combining the bound on $\|u\|_{L^{2}}$ given by (17.1), the mean-value theorem for definite integrals, and the Hölder continuity given by (7.2), we get the $L^{\infty}$ bound on $u$. A similar inequality holds for $m$. Next, we observe that Proposition 6.1 (see (6.2)) gives bounds for $\left\|u_{x x}\right\|_{L^{2}}$ and $\left\|m_{x x}\right\|_{L^{2}}$ of the same type of (7.1). Accordingly, the functions $u_{x}$ and $m_{x}$ are also $\frac{1}{2}$-Hölder continuous, and their $L^{\infty}$ norms are bounded by $\frac{C}{\sqrt{\epsilon}}$, where $C$ depends only on the constants in Assumptions 1 and 2 and on $\|V\|_{C^{2}}$.

Remark 7.3. Consider Problem 1 with $V$ replaced by $\lambda V$ for some $\lambda \in[0,1]$. By revisiting the proofs of Propositions 5.1 and 6.1 we can readily check that the bounds stated in these propositions are uniform with respect to $\lambda \in[0,1]$. More precisely, (5.1), (6.1), and (6.2) are still valid for a universal positive constant, $C$, that depends only on the constants in Assumptions 1 and 2 and on $\|V\|_{C^{2}}$. In particular, Proposition 7.2 remains unchanged. 


\section{Higher Regularity}

The bounds in the previous section give Hölder regularity for any solution $(u, m)$ of Problem 1 and for its derivatives $\left(u_{x}, m_{x}\right)$. Here, we use (2.1) to improve this result and prove Hölder regularity for $u_{x x}$ and $m_{x x}$.

Proposition 8.1. Suppose that Assumptions 15 hold. Let $(u, m)$ solve Problem 1 Then $(u, m) \in C^{2, \frac{1}{2}}(\mathbb{T}) \times C^{2, \frac{1}{2}}(\mathbb{T})$.

Proof. Solving for $m-m_{x x}$ in the second equation of (2.1) and replacing the resulting expression in the first equation yields

$$
\left[1+\epsilon^{2}+\epsilon H^{\prime \prime}\left(u_{x}\right) m\right] u_{x x}=\left(1+\epsilon^{2}\right) u+H\left(u_{x}\right)-\epsilon+V(x)-m^{\alpha}-\epsilon H^{\prime}\left(u_{x}\right) m_{x} .
$$

Because $H$ is convex, we have $H^{\prime \prime}\left(u_{x}\right) \geqslant 0$. Consequently, $1+\epsilon^{2}+\epsilon H^{\prime \prime}\left(u_{x}\right) m \geqslant 1>0$. This allows us to rewrite (8.1) as

$$
u_{x x}=\frac{\left(1+\epsilon^{2}\right) u+H\left(u_{x}\right)-\epsilon+V(x)-m^{\alpha}-\epsilon H^{\prime}\left(u_{x}\right) m_{x}}{1+\epsilon^{2}+\epsilon H^{\prime \prime}\left(u_{x}\right) m} .
$$

Because $u, m, u_{x}$, and $m_{x}$ are $\frac{1}{2}$-Hölder continuous and because $H$ and $H^{\prime}$ are locally Lipschitz functions, it follows that

$$
\left(1+\epsilon^{2}\right) u+H\left(u_{x}\right)-\epsilon+V(x)-m^{\alpha}-\epsilon H^{\prime}\left(u_{x}\right) m_{x}
$$

is also $\frac{1}{2}$-Hölder continuous. Similarly, due to Assumption [5] $1+\epsilon^{2}+\epsilon H^{\prime \prime}\left(u_{x}\right) m$ is also $\frac{1}{2}$-Hölder continuous and bounded from below. Therefore, $u_{x x}$ is $\frac{1}{2}$-Hölder continuous; thus, $u \in C^{2, \frac{1}{2}}(\mathbb{T})$.

Finally, we observe that the second equation in 2.1) is equivalent to

$$
m_{x x}=m+\epsilon\left(u-u_{x x}\right)-1-H^{\prime \prime}\left(u_{x}\right) m u_{x x}-H^{\prime}\left(u_{x}\right) m_{x} .
$$

Hence, analogous arguments to those used above yield that $m_{x x}$ is also $\frac{1}{2}$-Hölder continuous. Thus, $m \in C^{2, \frac{1}{2}}(\mathbb{T})$.

\section{Lower bounds on $m$}

Here, we establish our last a priori estimate, which gives lower bounds on $m$. We begin by proving an auxiliary result.

Lemma 9.1. Suppose that Assumptions 14, 4, and 17 hold. Let $(u, m)$ solve Problem 1. Then, there exists $\bar{\epsilon}_{0}>0$ such that for all $0<\epsilon<\bar{\epsilon}_{0}$, we have $\left\|\epsilon\left(u-u_{x x}\right)\right\|_{\infty}<\frac{1}{2}$.

Proof. We show that

$$
\lim _{\epsilon \rightarrow 0}\left\|\epsilon\left(u-u_{x x}\right)\right\|_{\infty}=0
$$

from which Lemma 9.1 easily follows.

To simplify the notation, in the remainder of this proof, $C$ represents a positive constant that is independent of $\epsilon$ and whose value may change from one instance to another.

By Proposition 7.2, we have that $\|u\|_{\infty} \leqslant C / \sqrt{\epsilon}$. Thus,

$$
\lim _{\epsilon \rightarrow 0}\|\epsilon u\|_{\infty}=0
$$
give

Next, we examine $\left\|\epsilon u_{x x}\right\|_{\infty}$. The identity (18.2) and the condition $1+\epsilon^{2}+\epsilon H^{\prime \prime}\left(u_{x}\right) m>1$

$$
\left\|\epsilon u_{x x}\right\|_{\infty} \leqslant\left\|\left(1+\epsilon^{2}\right) u\right\|_{\infty}+\left\|\epsilon H\left(u_{x}\right)\right\|_{\infty}+\epsilon^{2}+\|\epsilon V\|_{\infty}+\left\|\epsilon m^{\alpha}\right\|_{\infty}+\left\|\epsilon^{2} H^{\prime}\left(u_{x}\right) m_{x}\right\|_{\infty} .
$$


By (9.2) and by the boundedness of $V$, it follows that $\lim _{\epsilon \rightarrow 0}\left(\left\|\epsilon\left(1+\epsilon^{2}\right) u\right\|_{\infty}+\epsilon^{2}+\|\epsilon V\|_{\infty}\right)=$ 0 .

According to Propositions 5.1 and 6.1, we have that

$$
\int_{0}^{1} m^{\alpha+1} d x \leqslant C \text { and } \int_{0}^{1} \alpha m^{\alpha-1} m_{x}^{2} d x=\frac{4 \alpha}{(\alpha+1)^{2}} \int_{0}^{1}\left(m^{\frac{\alpha+1}{2}}\right)_{x}^{2} d x \leqslant C .
$$

The first integral guarantees that there exists $x_{0} \in \mathbb{T}$ such that $m^{\frac{\alpha+1}{2}}\left(x_{0}\right) \leqslant C$. Then, because $m>0$ and because $m \in C^{1}(\mathbb{T})$, the second integral together with Proposition 7.1 implies that for all $x \in \mathbb{T}$,

$$
0<m^{\alpha}(x)=\left(m^{\frac{\alpha}{2}}(x)\right)^{2} \leqslant\left(m^{\frac{\alpha+1}{2}}(x)-m^{\frac{\alpha+1}{2}}\left(x_{0}\right)+m^{\frac{\alpha+1}{2}}\left(x_{0}\right)+1\right)^{2} \leqslant C .
$$

Hence, $\lim _{\epsilon \rightarrow 0}\left\|\epsilon m^{\alpha}\right\|_{\infty}=0$.

Assumption 1 and Proposition 7.2 give

$$
\left|H\left(u_{x}\right)\right| \leqslant C\left(1+\epsilon^{-\frac{\gamma}{2}}\right) .
$$

This implies that $\lim _{\epsilon \rightarrow 0}\left\|\epsilon H\left(u_{x}\right)\right\|_{\infty}=0$ because $\gamma<2$ according to Assumption 6

Combining Assumption 7 with Proposition 7.2 gives the bound

$$
\left|H^{\prime}\left(u_{x}\right)\right| \leqslant C\left(1+\epsilon^{-\frac{\gamma-1}{2}}\right) .
$$

By Proposition [7.2, we have that $\left|m_{x}\right| \leqslant C / \sqrt{\epsilon}$. Therefore, invoking Assumption [6] once more, $\lim _{\epsilon \rightarrow 0}\left\|\epsilon^{2} H^{\prime}\left(u_{x}\right) m_{x}\right\|_{\infty}=0$.

Collecting all the limits proved above, we conclude from (9.3) that $\lim _{\epsilon \rightarrow 0}\left\|\epsilon u_{x x}\right\|_{\infty}=0$. This equality together with (9.2) proves (9.1).

Proposition 9.2. Suppose that Assumptions 1 4, 6, and 7 hold. Assume that $0<\epsilon<\bar{\epsilon}_{0}$, where $\bar{\epsilon}_{0}$ is determined by Lemma 9.1. Let $(u, m)$ solve Problem 1 . Then, there exists a constant $\bar{m}>0$ such that $m>\bar{m}$ in $\mathbb{T}$. Moreover, $\bar{m}$ is a universal constant depending only on the constants in Assumptions 1, 2, and 7 and on $\|V\|_{\infty}$.

Proof. Multiplying the second equation in (2.1) by $1 / m$ and integrating with respect to $x$ in $[0,1]$, we obtain

$$
\int_{0}^{1}\left(1-\frac{m_{x x}}{m}-\frac{\left(H^{\prime}\left(u_{x}\right) m\right)_{x}}{m}\right) d x=\int_{0}^{1}\left(\frac{1}{m}-\epsilon \frac{u-u_{x x}}{m}\right) d x .
$$

Integration by parts and periodicity yields

$$
\int_{0}^{1} \frac{m_{x x}}{m} d x=\int_{0}^{1} \frac{m_{x}^{2}}{m^{2}} d x
$$

Then, 9.4 can be rewritten as

$$
\int_{0}^{1}\left(\frac{1}{m}+\frac{m_{x}^{2}}{m^{2}}\right) d x=1+\int_{0}^{1} \frac{\epsilon\left(u-u_{x x}\right)}{m} d x-\int_{0}^{1} \frac{\left(H^{\prime}\left(u_{x}\right) m\right)_{x}}{m} d x .
$$

Next, we estimate the right-hand side of this identity. By Lemma 9.1, for $0<\epsilon<\bar{\epsilon}_{0}$, we have $\left\|\epsilon\left(u-u_{x x}\right)\right\|_{\infty}<1 / 2$. Consequently,

$$
\int_{0}^{1}\left(\frac{1}{2 m}+\frac{m_{x}^{2}}{m^{2}}\right) d x \leqslant 1+\left|\int_{0}^{1} \frac{\left(H^{\prime}\left(u_{x}\right) m\right)_{x}}{m} d x\right|=1+\left|\int_{0}^{1} H^{\prime}\left(u_{x}\right) \frac{m_{x}}{m} d x\right|,
$$

where in the last equality we use the integration by parts formula and the periodicity of $u_{x}$. In view of Cauchy's inequality, we conclude that

$$
\left|\int_{0}^{1} H^{\prime}\left(u_{x}\right) \frac{m_{x}}{m} d x\right| \leqslant \int_{0}^{1}\left|H^{\prime}\left(u_{x}\right) \frac{m_{x}}{m}\right| d x \leqslant \int_{0}^{1}\left(\frac{\left(H^{\prime}\left(u_{x}\right)\right)^{2}}{2}+\frac{m_{x}^{2}}{2 m^{2}}\right) d x .
$$


Invoking Assumptions 6 and 7 we obtain the estimates

$$
\left(H^{\prime}\left(u_{x}\right)\right)^{2} \leqslant \bar{C}^{2}\left(1+\left|u_{x}\right|^{\gamma-1}\right)^{2} \leqslant 2 \bar{C}^{2}\left(1+\left|u_{x}\right|^{2(\gamma-1)}\right) \leqslant 2 \bar{C}^{2}\left(2+\left|u_{x}\right|^{2}\right)
$$

in $\mathbb{T}$. These estimates, (9.5), (9.6), and Proposition 5.1 yield

$$
\int_{0}^{1}\left(\frac{1}{2 m}+\frac{m_{x}^{2}}{2 m^{2}}\right) d x \leqslant 1+\bar{C}^{2}\left(2+\frac{C}{\epsilon}\right) .
$$

Consequently, for $\tilde{C}=2+2 \bar{C}^{2}\left(2+\frac{C}{\epsilon}\right)$, we obtain the two following bounds

$$
\int_{0}^{1} \frac{1}{m} d x \leqslant \tilde{C} \text { and } \int_{0}^{1} \frac{m_{x}^{2}}{m^{2}} d x=\int_{0}^{1}(\ln (m))_{x}^{2} d x \leqslant \tilde{C} .
$$

The first bound implies that there exists $x_{0} \in \mathbb{T}$ such that $\frac{1}{m\left(x_{0}\right)} \leqslant \tilde{C}+1$; that is, $\ln \left(m\left(x_{0}\right)\right) \geqslant$ $-\ln (\tilde{C}+1)$. The second bound, together with Proposition 7.1 implies that for all $x \in \mathbb{T}$, $\left|\ln (m(x))-\ln \left(m\left(x_{0}\right)\right)\right| \leqslant \sqrt{\tilde{C}}$. Hence, for all $x \in \mathbb{T}$,

$$
m(x) \geqslant e^{-\sqrt{\tilde{C}}-\ln (\tilde{C}+1)},
$$

which completes the proof.

Remark 9.3. As in Remark 7.3, the statement of Proposition 9.2 remains unchanged if we replace $V$ by $\lambda V$ for some $\lambda \in[0,1]$ in Problem 1 .

\section{The linearized operator}

Consider the functional, $F$, defined for $(u, m, \lambda) \in C^{2, \frac{1}{2}}(\mathbb{T}) \times C^{2, \frac{1}{2}}(\mathbb{T} ;] 0, \infty[) \times[0,1]$ by

$$
F(u, m, \lambda)=\left[\begin{array}{c}
u-u_{x x}+H\left(u_{x}\right)+\lambda V-m^{\alpha}-\epsilon\left(m-m_{x x}\right) \\
m-m_{x x}-\left(H^{\prime}\left(u_{x}\right) m\right)_{x}-1+\epsilon\left(u-u_{x x}\right)
\end{array}\right] .
$$

Note that under Assumption 5 , the functional $F$ is a $C^{1}$ map between $C^{2, \frac{1}{2}}(\mathbb{T}) \times C^{2, \frac{1}{2}}(\mathbb{T} ;] 0, \infty[) \times$ $[0,1]$ and $C^{0, \frac{1}{2}}(\mathbb{T}) \times C^{2, \frac{1}{2}}(\mathbb{T})$.

To prove Theorem 2.1 we use the continuation method and show that for every $\lambda \in[0,1]$, the equation

$$
F(u, m, \lambda)=0
$$

has a solution, $(u, m) \in C^{2, \frac{1}{2}}(\mathbb{T}) \times C^{2, \frac{1}{2}}(\mathbb{T} ;] 0, \infty[)$. Theorem 2.1 then follows by taking $\lambda=1$ and by observing that system (2.1) is equivalent to $F(u, m, 1)=0$.

The implicit function theorem plays a crucial role in proving the solvability of (10.2). To use this theorem, for each $\lambda \in[0,1]$, we introduce the linearized operator $L$ of $F(\cdot, \cdot, \lambda)$ at $(u, m) \in C^{2, \frac{1}{2}}(\mathbb{T}) \times C^{2, \frac{1}{2}}(\mathbb{T} ;] 0, \infty[)$; that is,

$$
\begin{aligned}
L(f, v) & =\left.\frac{\partial F}{\partial \mu}(u+\mu v, m+\mu f, \lambda)\right|_{\mu=0} \\
& =\left[\begin{array}{c}
v-v_{x x}+H^{\prime}\left(u_{x}\right) v_{x}-\alpha m^{\alpha-1} f-\epsilon\left(f-f_{x x}\right) \\
f-f_{x x}-\left(H^{\prime \prime}\left(u_{x}\right) v_{x} m+H^{\prime}\left(u_{x}\right) f\right)_{x}+\epsilon\left(v-v_{x x}\right)
\end{array}\right]
\end{aligned}
$$

for $(f, v) \in C^{2, \frac{1}{2}}(\mathbb{T}) \times C^{2, \frac{1}{2}}(\mathbb{T})$. Under Assumption 5 and because $(u, m) \in C^{2, \frac{1}{2}}(\mathbb{T}) \times$ $C^{2, \frac{1}{2}}(\mathbb{T} ;] 0, \infty[), L$ defines a map from $C^{2, \frac{1}{2}}(\mathbb{T}) \times C^{2, \frac{1}{2}}(\mathbb{T})$ into $C^{0, \frac{1}{2}}(\mathbb{T}) \times C^{0, \frac{1}{2}}(\mathbb{T})$. Moreover, this map is continuous and linear. Next, we show that it is also an isomorphism between $C^{2, \frac{1}{2}}(\mathbb{T}) \times C^{2, \frac{1}{2}}(\mathbb{T})$ and $C^{0, \frac{1}{2}}(\mathbb{T}) \times C^{0, \frac{1}{2}}(\mathbb{T})$.

Proposition 10.1. Suppose that Assumptions 4 and $[5$ hold. Fix $\lambda \in[0,1]$ and assume that $(u, m) \in C^{2, \frac{1}{2}}(\mathbb{T}) \times C^{2, \frac{1}{2}}(\mathbb{T} ;] 0, \infty[)$ satisfies $F(u, m, \lambda)=0$. Then, the operator, $L$, given by (10.3) is an isomorphism between $C^{2, \frac{1}{2}}(\mathbb{T}) \times C^{2, \frac{1}{2}}(\mathbb{T})$ and $C^{0, \frac{1}{2}}(\mathbb{T}) \times C^{0, \frac{1}{2}}(\mathbb{T})$. 
Proof. To prove the proposition, we begin by applying the Lax-Milgram theorem in $H^{1}(\mathbb{T}) \times$ $H^{1}(\mathbb{T})$, after which we bootstrap additional regularity. Here, we endow $H^{1}(\mathbb{T}) \times H^{1}(\mathbb{T})$ with the inner product

$$
\left\langle\left(\theta_{1}, \theta_{2}\right),\left(\bar{\theta}_{1}, \bar{\theta}_{2}\right)\right\rangle_{H^{1}(\mathbb{T}) \times H^{1}(\mathbb{T})}=\int_{0}^{1}\left(\theta_{1} \bar{\theta}_{1}+\theta_{2} \bar{\theta}_{2}+\theta_{1 x} \bar{\theta}_{1 x}+\theta_{2 x} \bar{\theta}_{2 x}\right) d x
$$

for $\left(\theta_{1}, \theta_{2}\right),\left(\bar{\theta}_{1}, \bar{\theta}_{2}\right) \in H^{1}(\mathbb{T}) \times H^{1}(\mathbb{T})$.

Consider the bilinear form $B:\left(H^{1}(\mathbb{T}) \times H^{1}(\mathbb{T})\right) \times\left(H^{1}(\mathbb{T}) \times H^{1}(\mathbb{T})\right) \rightarrow \mathbb{R}$ defined for $(v, f),\left(w_{1}, w_{2}\right) \in H^{1}(\mathbb{T}) \times H^{1}(\mathbb{T})$ by

$$
\begin{aligned}
B\left(\left(\begin{array}{l}
v \\
f
\end{array}\right),\left(\begin{array}{l}
w_{1} \\
w_{2}
\end{array}\right)\right)= & \int_{0}^{1}(f+\epsilon v) w_{1} d x+\int_{0}^{1}\left[f_{x}+H^{\prime \prime}\left(u_{x}\right) v_{x} m+H^{\prime}\left(u_{x}\right) f+\epsilon v_{x}\right] w_{1 x} d x \\
& -\int_{0}^{1}\left[v+H^{\prime}\left(u_{x}\right) v_{x}-\alpha m^{\alpha-1} f-\epsilon f\right] w_{2} d x+\int_{0}^{1}\left(\epsilon f_{x}-v_{x}\right) w_{2 x} d x .
\end{aligned}
$$

Note that if $(v, f) \in C^{2, \frac{1}{2}}(\mathbb{T}) \times C^{2, \frac{1}{2}}(\mathbb{T})$, then

$$
B\left(\left(\begin{array}{l}
v \\
f
\end{array}\right),\left(\begin{array}{l}
w_{1} \\
w_{2}
\end{array}\right)\right)=\int_{0}^{1}\left[-L_{1}(f, v) w_{2}+L_{2}(f, v) w_{1}\right] d x,
$$

where $L_{1}$ and $L_{2}$ are the first and second components of $L$, respectively.

Next, we prove that $B$ is coercive and bounded in $H^{1}(\mathbb{T}) \times H^{1}(\mathbb{T})$. Fix $(v, f),\left(w_{1}, w_{2}\right) \in$ $H^{1}(\mathbb{T}) \times H^{1}(\mathbb{T})$. Using the integration by parts formula and the periodicity of $v$ and $f$, we obtain

$$
B\left(\left(\begin{array}{l}
v \\
f
\end{array}\right),\left(\begin{array}{l}
v \\
f
\end{array}\right)\right)=\int_{0}^{1}\left[\alpha m^{\alpha-1} f^{2}+H^{\prime \prime}\left(u_{x}\right) v_{x}^{2} m+\epsilon\left(v^{2}+v_{x}^{2}+f^{2}+f_{x}^{2}\right)\right] d x .
$$

Because $H^{\prime \prime} \geqslant 0$ by Assumption 4 and because $m>0$, we have that

$$
B\left(\left(\begin{array}{l}
v \\
f
\end{array}\right),\left(\begin{array}{l}
v \\
f
\end{array}\right)\right) \geqslant \epsilon\left\|\left(\begin{array}{l}
v \\
f
\end{array}\right)\right\|_{H^{1}(\mathbb{T}) \times H^{1}(\mathbb{T})}^{2},
$$

which proves the coercivity of $B$.

Because $m, u$, and $H$ are $C^{2, \frac{1}{2}}$-functions on the compact set [0,1], we have that $m, u, m_{x}$, $u_{x}, u_{x x}, H, H^{\prime}\left(u_{x}\right)$, and $H^{\prime \prime}\left(u_{x}\right)$ are bounded. Therefore, there exists a positive constant, $C$, that depends only on these bounds and for which

$$
\left|B\left(\left(\begin{array}{l}
v \\
f
\end{array}\right),\left(\begin{array}{l}
w_{1} \\
w_{2}
\end{array}\right)\right)\right| \leqslant C\left\|\left(\begin{array}{l}
v \\
f
\end{array}\right)\right\|_{H^{1}(\mathbb{T}) \times H^{1}(\mathbb{T})}\left\|\left(\begin{array}{l}
w_{1} \\
w_{2}
\end{array}\right)\right\|_{H^{1}(\mathbb{T}) \times H^{1}(\mathbb{T})},
$$

where we also used Hölder's inequality. This proves the boundedness of $B$.

Finally, we fix $b=\left(b_{1}, b_{2}\right) \in C^{0, \frac{1}{2}}(\mathbb{T}) \times C^{0, \frac{1}{2}}(\mathbb{T})$, and we consider the bounded and linear functional $G: H^{1}(\mathbb{T}) \times H^{1}(\mathbb{T}) \rightarrow \mathbb{R}$ defined for $\left(w_{1}, w_{2}\right) \in H^{1}(\mathbb{T}) \times H^{1}(\mathbb{T})$ by

$$
G\left(\begin{array}{l}
w_{1} \\
w_{2}
\end{array}\right)=\int_{0}^{1}\left(-b_{1} w_{2}+b_{2} w_{1}\right) d x .
$$

By the Lax-Milgram theorem, there exists a unique $(v, f) \in H^{1}(\mathbb{T}) \times H^{1}(\mathbb{T})$ such that for all $\left(w_{1}, w_{2}\right) \in H^{1}(\mathbb{T}) \times H^{1}(\mathbb{T})$, we have

$$
B\left(\left(\begin{array}{l}
v \\
f
\end{array}\right),\left(\begin{array}{l}
w_{1} \\
w_{2}
\end{array}\right)\right)=G\left(\begin{array}{l}
w_{1} \\
w_{2}
\end{array}\right) .
$$

This is equivalent to saying that for all $\left(w_{1}, w_{2}\right) \in H^{1}(\mathbb{T}) \times H^{1}(\mathbb{T})$,

$$
B\left(\left(\begin{array}{c}
v \\
f
\end{array}\right),\left(\begin{array}{c}
-w_{2} \\
w_{1}
\end{array}\right)\right)=G\left(\begin{array}{c}
-w_{2} \\
w_{1}
\end{array}\right)=\int_{0}^{1}\left(-b_{1} w_{1}-b_{2} w_{2}\right) d x .
$$


From this and (10.4), we conclude that $L(f, v)=b$ has a unique weak solution $(f, v) \in$ $H^{1}(\mathbb{T}) \times H^{1}(\mathbb{T})$. Because $b \in C^{0, \frac{1}{2}}(\mathbb{T}) \times C^{0, \frac{1}{2}}(\mathbb{T})$ is arbitrary, $L$ is injective. To prove surjectivity, it suffices to check that the weak solution of $L(f, v)=b$ is in $C^{2, \frac{1}{2}}(\mathbb{T}) \times C^{2, \frac{1}{2}}(\mathbb{T})$. This higher regularity follows from a bootstrap argument.

Fix $b=\left(b_{1}, b_{2}\right) \in C^{0, \frac{1}{2}}(\mathbb{T}) \times C^{0, \frac{1}{2}}(\mathbb{T})$ and let $(f, v) \in H^{1}(\mathbb{T}) \times H^{1}(\mathbb{T})$ be the weak solution of $L(f, v)=b$ given by the Lax-Milgram theorem. Then, we have the following identity in the weak sense:

$$
v_{x x}=\frac{g}{1+\epsilon^{2}+\epsilon H^{\prime \prime}\left(u_{x}\right) m},
$$

where $g=v\left(1+\epsilon^{2}\right)+H^{\prime}\left(u_{x}\right) v_{x}-\alpha m^{\alpha-1} f-\epsilon v_{x}\left(H^{\prime}\left(u_{x}\right) m\right)_{x}-\epsilon\left(H^{\prime}\left(u_{x}\right) f\right)_{x}-\epsilon b_{2}-b_{1} \in L^{2}(\mathbb{T})$. We recall that $1+\epsilon^{2}+\epsilon H^{\prime \prime}\left(u_{x}\right) m>1$. Hence, $v_{x x} \in L^{2}(\mathbb{T})$, and so $v \in H^{2}(\mathbb{T})$. Moreover, because

$$
f_{x x}=f-\left(H^{\prime \prime}\left(u_{x}\right) v_{x} m\right)_{x}-\left(H^{\prime}\left(u_{x}\right) f\right)_{x}+\epsilon\left(v-v_{x x}\right)-b_{2}
$$

in the weak sense, similar arguments yield $f_{x x} \in L^{2}(\mathbb{T})$ and $f \in H^{2}(\mathbb{T})$.

So far, $(f, v) \in C^{1, \frac{1}{2}}(\mathbb{T}) \times C^{1, \frac{1}{2}}(\mathbb{T})$. This implies that $g \in C^{0, \frac{1}{2}}(\mathbb{T})$. Then, using the fact that $1+\epsilon^{2}+\epsilon H^{\prime \prime}\left(u_{x}\right) m$ also belongs to $C^{0, \frac{1}{2}}(\mathbb{T})$ and is bounded from below by 1 , from (10.5) it follows that $v_{x x} \in C^{0, \frac{1}{2}}(\mathbb{T})$. Consequently, in view of (10.6), $f_{x x} \in C^{0, \frac{1}{2}}(\mathbb{T})$. Hence, $(f, v) \in C^{2, \frac{1}{2}}(\mathbb{T}) \times C^{2, \frac{1}{2}}(\mathbb{T})$. Therefore, the unique solution given by the Lax-Milgram theorem is a strong solution with $C^{2, \frac{1}{2}}$ regularity. Thus, $L$ is surjective. Because $L$ is injective and surjective, it is an isomorphism.

\section{Proof of the Main Theorem}

In this last section, we prove Theorem 2.1. We assume that $\epsilon>0$ satisfies $\epsilon<\min \left\{1, \epsilon_{0}, \bar{\epsilon}_{0}\right\}$, where $\epsilon_{0}$ and $\bar{\epsilon}_{0}$ are given by Proposition 4.1 and Lemma 9.1 , respectively.

Let $F$ be the functional defined in (10.1). For each $\lambda \in[0,1]$, consider the problem of finding $(u, m) \in C^{2, \frac{1}{2}}(\mathbb{T}) \times C^{2, \frac{1}{2}}(\mathbb{T} ;] 0, \infty[)$ satisfying (10.2). From Propositions 4.1 and 8.1. such a pair $(u, m)$ exists for $\lambda=0$. Next, using the continuation method, we prove that this is true not only for $\lambda=0$ but also for all $\lambda \in[0,1]$.

More precisely, let $\Lambda$ be the set of values, $\lambda \in[0,1]$, for which equation (10.2) has a solution $(u, m) \in C^{2, \frac{1}{2}}(\mathbb{T}) \times C^{2, \frac{1}{2}}(\mathbb{T})$ with $m \geqslant \bar{m}$ in $\mathbb{T}$, where $\bar{m}>0$ is given by Proposition 9.2. Note that $\bar{m}$ does not depend on $\lambda$ (see Remark 9.3). As we just argued, $\Lambda$ is a nonempty set. In the subsequent two propositions, we show that $\Lambda$ is a closed and open subset of $[0,1]$. Consequently, $\Lambda=[0,1]$.

Proposition 11.1. Suppose that Assumptions 17 hold. Then, $\Lambda$ is a closed subset of $[0,1]$.

Proof. Let $\left(\lambda^{n}\right)_{n \in \mathbb{N}} \subset \Lambda$ and $\lambda \in[0,1]$ be such that $\lim _{n \rightarrow \infty} \lambda^{n}=\lambda$. We claim that $\lambda \in \Lambda$.

By definition of $\Lambda$, for each $n \in \mathbb{N}$, there exists $\left(u^{n}, m^{n}\right) \in C^{2, \frac{1}{2}}(\mathbb{T}) \times C^{2, \frac{1}{2}}(\mathbb{T})$ satisfying (10.2) and $m^{n} \geqslant \bar{m}$ in $\mathbb{T}$. Then, by Proposition 7.2 (also see Remark 7.3 ) $,\left(u^{n}\right)_{n \in \mathbb{N}},\left(m^{n}\right)_{n \in \mathbb{N}}$, $\left(u_{x}^{n}\right)_{n \in \mathbb{N}}$, and $\left(m_{x}^{n}\right)_{n \in \mathbb{N}}$ are uniformly bounded in $C^{0, \frac{1}{2}}(\mathbb{T})$. Consequently, by the ArzeláAscoli theorem, we can find $(u, m, \tilde{u}, \tilde{m}) \in C^{0, \frac{1}{2}}(\mathbb{T}) \times C^{0, \frac{1}{2}}(\mathbb{T}) \times C^{0, \frac{1}{2}}(\mathbb{T}) \times C^{0, \frac{1}{2}}(\mathbb{T})$ such that, up to a subsequence that we do not relabel,

$$
\lim _{n \rightarrow \infty}\left\|\left(u^{n}, m^{n}, u_{x}^{n}, m_{x}^{n}\right)-(u, m, \tilde{u}, \tilde{m})\right\|_{\infty}=0 .
$$

We now recall that if $\left(w^{n}\right)_{n \in \mathbb{N}}$ is a sequence of differentiable functions on $[0,1]$ such that $\left(w^{n}\right)_{n \in \mathbb{N}}$ converges uniformly to some $w$ on $[0,1]$ and such that $\left(w_{x}^{n}\right)_{n \in \mathbb{N}}$ converges uniformly on $[0,1]$, then $w_{x}=\lim _{n \rightarrow \infty} w_{x}^{n}$ on $[0,1]$. Consequently, by (11.1), we have that $\tilde{u}=u_{x}$ and $\tilde{m}=m_{x}$.

Next, we show that $\left(u_{x x}^{n}\right)_{n \in \mathbb{N}}$ and $\left(m_{x x}^{n}\right)_{n \in \mathbb{N}}$ are also uniformly convergent sequences on $[0,1]$. In view of (8.2), we have that, for every $n \in \mathbb{N}$,

$$
u_{x x}^{n}=\frac{\left(1+\epsilon^{2}\right) u^{n}+H\left(u_{x}^{n}\right)-\epsilon+\lambda^{n} V(x)-\left(m^{n}\right)^{\alpha}-\epsilon H^{\prime}\left(u_{x}^{n}\right) m_{x}^{n}}{1+\epsilon^{2}+\epsilon H^{\prime \prime}\left(u_{x}^{n}\right) m^{n}} .
$$


By Assumption 5 and by the uniform convergence of $\left(u^{n}, m^{n}, \lambda^{n}, u_{x}^{n}, m_{x}^{n}\right)_{n \in \mathbb{N}}$ to $\left(u, m, \lambda, u_{x}, m_{x}\right)$ on $[0,1]$, it follows from (11.2) that $\left(u_{x x}^{n}\right)_{n \in \mathbb{N}}$ converges uniformly on $[0,1]$. Then, the limit of $\left(u_{x x}^{n}\right)_{n \in \mathbb{N}}$ is necessarily $u_{x x}$. Analogous arguments (see (8.3)) give that $\left(m_{x x}^{n}\right)_{n \in \mathbb{N}}$ converges uniformly to $m_{x x}$ on $[0,1]$. Consequently, $(u, m) \in C^{2, \frac{1}{2}}(\mathbb{T}) \times C^{2, \frac{1}{2}}(\mathbb{T} ;] 0, \infty[)$. Moreover, $\lim _{n \rightarrow \infty} F\left(u^{n}, m^{n}, \lambda^{n}\right)=F(u, m, \lambda)$. Finally, because for all $n \in \mathbb{N}, F\left(u^{n}, m^{n}, \lambda^{n}\right)=0$ and $m^{n} \geqslant \bar{m}$ in $\mathbb{T}$, we have that $F(u, m, \lambda)=0$ and $m \geqslant \bar{m}$ in $\mathbb{T}$. Thus, $\lambda \in \Lambda$. This completes the proof.

Proposition 11.2. Suppose that Assumptions 10 hold. Then, $\Lambda$ is an open subset of $[0,1]$.

Proof. Let $\lambda_{0} \in \Lambda$. Then, there exists $\left(u_{0}, m_{0}\right) \in C^{2, \frac{1}{2}}(\mathbb{T}) \times C^{2, \frac{1}{2}}(\mathbb{T})$ satisfying $F\left(u_{0}, m_{0}, \lambda_{0}\right)=$ 0 and $m_{0} \geqslant \bar{m}$ in $\mathbb{T}$. By Proposition 10.1 and by the implicit function theorem in Banach spaces (see, for example, $\left[3\right.$ ), we can find $\delta>0$ such that, for every $\left.\lambda^{*} \in\right] \lambda-\lambda_{0}, \lambda+\lambda_{0}[$, there exists $\left(u^{*}, m^{*}\right) \in C^{2, \frac{1}{2}}(\mathbb{T}) \times C^{2, \frac{1}{2}}(\mathbb{T})$ satisfying $F\left(u^{*}, m^{*}, \lambda^{*}\right)=0$ and $m^{*} \geqslant \bar{m}$ in $\mathbb{T}$. Moreover, the implicit function theorem also guarantees that the map $\lambda^{*} \mapsto m^{*}$ is continuous. Hence, if $\delta$ is small enough, we have $m^{*}>0$ in $\mathbb{T}$. Then, Proposition 9.1 gives $m^{*}>\bar{m}$ in $\mathbb{T}$. Therefore, $\lambda^{*} \in \Lambda$ and, consequently, $\Lambda$ is open.

Finally, we sum up the proof of our main result.

Proof of Theorem 2.1. Let $\epsilon>0$ be such that $\epsilon<\min \left\{1, \epsilon_{0}, \bar{\epsilon}_{0}\right\}$, where $\epsilon_{0}$ is given by Proposition 4.1 and where $\bar{\epsilon}_{0}$ is given by Lemma 9.1 .

Propositions 11.1 and 11.2 give that $\Lambda$ is a relatively open and closed set in $[0,1]$. It is a non-empty set due to Propositions 4.1, 8.1, and 9.2. Hence, $\Lambda=[0,1]$. Finally, we observe that Theorem 2.1 corresponds to the $\lambda=1$ case.

\section{Acknowledgements}

This summer camp would not have been possible without major help and support from KAUST. David Yeh and his team at the Visiting Student Research Program did a fantastic job in organizing all the logistics. In addition, the CEMSE Division provided valuable additional support. Finally, we would like to thank the faculty, research scientists, postdocs and Ph.D. students who, in the first three weeks of the semester, made time to give courses, lectures, and work with the students.

\section{References}

[1] P. Cardaliaguet, P. Garber, A. Porretta, and D. Tonon. Second order mean field games with degenerate diffusion and local coupling. Preprint, 2014.

[2] M. Cirant. Multi-population Mean Field Games systems with Neumann boundary conditions. J. Math. Pures Appl. (9), 103(5):1294-1315, 2015.

[3] J. Dieudonné. Foundations of modern analysis. Vol. I, Academic Press, New York, 1969.

[4] L. C. Evans. Partial Differential Equations. Graduate Studies in Mathematics. American Mathematical Society, 1998.

[5] R. Ferreira and D. Gomes. Existence of weak solutions for stationary mean-field games through weak solutions. Preprint.

[6] D. Gomes and H. Mitake. Stationary mean-field games with congestion and quadratic Hamiltonians. Preprint.

[7] D. Gomes and S. Patrizi. Obstacle mean-field game problem. To appear in Interfaces and Free Boundaries, 2013. 
[8] D. Gomes, S. Patrizi, and V. Voskanyan. On the existence of classical solutions for stationary extended mean field games. Nonlinear Anal., 99:49-79, 2014.

[9] D. Gomes and E. Pimentel. Local regularity for mean-field games in the whole space. Preprint.

[10] D. Gomes and E. Pimentel. Time dependent mean-field games with logarithmic nonlinearities. Preprint.

[11] D. Gomes, E. Pimentel, and H Sanchez-Morgado. Time dependent mean-field games in the superquadratic case. Preprint, 2013.

[12] D. Gomes, E. A. Pimentel, and H. Sánchez-Morgado. Time-dependent mean-field games in the subquadratic case. Comm. Partial Differential Equations, 40(1):40-76, 2015.

[13] D. Gomes, G. E. Pires, and H. Sánchez-Morgado. A-priori estimates for stationary mean-field games. Netw. Heterog. Media, 7(2):303-314, 2012.

[14] D. Gomes and R. Ribeiro. Mean field games with logistic population dynamics. 52nd IEEE Conference on Decision and Control (Florence, December 2013), 2013.

[15] D. Gomes and H. Sánchez Morgado. A stochastic Evans-Aronsson problem. Trans. Amer. Math. Soc., 366(2):903-929, 2014.

[16] D. Gomes and J. Saúde. Mean field games models - a brief survey. Dyn. Games Appl., 4(2):110-154, 2014.

[17] J. Graber. Weak solutions for mean field games with congestion. Preprint.

[18] M. Huang, P. E. Caines, and R. P. Malhamé. Large-population cost-coupled LQG problems with nonuniform agents: individual-mass behavior and decentralized $\epsilon$-Nash equilibria. IEEE Trans. Automat. Control, 52(9):1560-1571, 2007.

[19] M. Huang, R. P. Malhamé, and P. E. Caines. Large population stochastic dynamic games: closed-loop McKean-Vlasov systems and the Nash certainty equivalence principle. Commun. Inf. Syst., 6(3):221-251, 2006.

[20] J.-M. Lasry and P.-L. Lions. Jeux à champ moyen. I. Le cas stationnaire. C. R. Math. Acad. Sci. Paris, 343(9):619-625, 2006.

[21] J.-M. Lasry and P.-L. Lions. Jeux à champ moyen. II. Horizon fini et contrôle optimal. C. R. Math. Acad. Sci. Paris, 343(10):679-684, 2006.

[22] J.-M. Lasry and P.-L. Lions. Mean field games. Jpn. J. Math., 2(1):229-260, 2007.

[23] P.-L. Lions. College de France course on mean-field games. 2007-2011.

[24] P.-L. Lions. IMA, University of Minessota. Course on mean-field games. Video. http://www.ima.umn.edu/2012-2013/sw11.12-13.12/. 2012.

[25] E. Pimentel and V. Voskanyan. Regularity for second-order stationaty mean-field games. Preprint.

[26] A. Porretta. On the planning problem for the mean-field games system. Dyn. Games Appl., 2013.

[27] A. Porretta. Weak Solutions to Fokker-Planck Equations and Mean Field Games. Arch. Ration. Mech. Anal., 216(1):1-62, 2015. 